\title{
Soft x-ray holographic microscopy
}

\author{
Daniel Stickler, ${ }^{1}$ Robert Frömter, ${ }^{1, a)}$ Holger Stillrich, ${ }^{1}$ Christian Menk, ${ }^{1}$ Carsten Tieg, ${ }^{2}$ \\ Simone Streit-Nierobisch, ${ }^{3}$ Michael Sprung, ${ }^{3}$ Christian Gutt, ${ }^{3}$ Lorenz-M. Stadler, ${ }^{3}$ \\ Olaf Leupold, ${ }^{3}$ Gerhard Grübel, ${ }^{3}$ and Hans Peter Oepen ${ }^{1}$ \\ ${ }^{1}$ Institut für Angewandte Physik, Universität Hamburg, Jungiusstr. 11, 20355 Hamburg, Germany \\ ${ }^{2}$ European Synchrotron Radiation Facility (ESRF), B.P. 200, F-38043 Grenoble Cedex, France \\ ${ }^{3}$ Deutsches Elektronen-Synchrotron (DESY), Notkestr. 85, 22607 Hamburg, Germany
}

(Received 21 September 2009; accepted 9 December 2009; published online 25 January 2010)

\begin{abstract}
We present a new x-ray microscopy technique based on Fourier transform holography (FTH), where the sample is separate from the optics part of the setup. The sample can be shifted with respect to the holography optics, thus large-scale or randomly distributed objects become accessible. As this extends FTH into a true microscopy technique, we call it x-ray holographic microscopy (XHM). FTH allows nanoscale imaging without the need for nanometer-size beams. Simple Fourier transform yields an unambiguous image reconstruction. We demonstrate XHM by studying the magnetic domain evolution of a $\mathrm{Co} / \mathrm{Pt}$ multilayer film as function of locally varied iron overlayer thickness. () 2010 American Institute of Physics. [doi:10.1063/1.3291942]
\end{abstract}

The ultimate $\mathrm{x}$-ray microscope provides a resolution that is only limited by the wavelength of the radiation, it has a large field of view, and it allows positioning arbitrarily connected objects in the beam. The image formation process should be simple, unambiguous, and free of artifacts. Scanning transmission $\mathrm{x}$-ray microscopy (STXM) provides a scanning mode with a straightforward data analysis. The resolution of STXM, however, is limited by the spot size on the sample. Coherent diffractive imaging (CDI), on the contrary, promises to reach diffraction-limited resolution by measuring oversampled diffraction patterns. ${ }^{1}$ The demanding image reconstruction and the need for isolated objects, however, prevent a widespread and easy-to-use application of the method. Recently, two approaches have been demonstrated that enable CDI investigation of nonisolated objects. Ptychography ${ }^{2}$ uses multiple diffraction patterns from overlapping sample areas, that are reconstructed jointly by applying a complex iterative phase retrieval algorithm. Keyhole CDI (Ref. 3) uses a single diffraction pattern from a sharply defined, divergent beam to reconstruct a section of an extended object. However, phase retrieval algorithms without any prior knowledge of the sample often yield slow convergence and ambiguity in the image formation process.

Much simpler is the image formation process using Fourier transform holography (FTH), ${ }^{4}$ where the scattered radiation from the sample interferes with a reference wave and forms a hologram on the detector. Reverse Fourier transform of the measured diffraction pattern yields an unambiguous image of the object. As the phases are encoded in the hologram, several numerical contrast enhancing procedures, as e.g., Zernike or Schlieren phase contrast, can be applied to the image. ${ }^{5}$ The spatial resolution in FTH-based methods is limited by the size of the reference aperture-today FTH masks can be routinely produced with reference holes of 30 $\mathrm{nm}$ size. However, the image obtained by reverse Fourier transform provides an excellent starting point for a further phase retrieval treatment. In such a way the resolution limitation of FTH can be overcome. ${ }^{6}$ FTH is especially attractive

${ }^{a)}$ Electronic mail: rfroemte@ physik.uni-hamburg.de. in the soft x-ray regime where the photon energy can be tuned to element-specific core level energies allowing for element-specific contrast in the images. This can be used for example to image magnetic domain structures ${ }^{4,7}$ using X-ray magnetic circular dichroism (XMCD) or for anomalous diffraction imaging. ${ }^{8}$ Extensions to multiple reference scatterers $^{9}$ and more elaborate reference signals ${ }^{10}$ to increase the signal-to-noise ratio without sacrifice in spatial resolution can be easily integrated into the XHM setup.

So far, the need for a reference wave close to the object appeared to impose severe restrictions on the design: The FTH optics (semitransparent object hole and holes for the reference waves) have been physically integrated with the sample, i.e., the optical FTH mask has been prepared directly into an opaque metal film on a transparent $\mathrm{Si}_{3} \mathrm{~N}_{4}$ membrane that carried the sample on the other side. The reference holes were created by milling nanometer sized channels through the whole stack. This setup locks the field of view to a $2 \mu \mathrm{m}$ sized sample area, without the possibility to investigate other areas on the sample. Much larger object holes are not feasible, as the transverse coherence length $\xi_{\mathrm{t}}$ of the x-ray beam limits the lateral extent of the structure (object hole and references) on the holography mask. In addition, the signal-tonoise ratio at fixed reference hole size (resolution) scales inversely to the diameter of the object hole and will thus decrease accordingly. ${ }^{11}$

Our new concept is to separate the sample from the optical elements using two $\mathrm{Si}_{3} \mathrm{~N}_{4}$ membranes. A sketch of the setup, as mounted in the vacuum system, is shown in Fig. 1. The upstream membrane contains the FTH mask, i.e., the object and the reference holes arranged in a conventional FTH geometry. This membrane has been initially covered with an opaque $800 \mathrm{~nm}$ thick gold film. Both, the object hole and one or more reference holes are fabricated by focused ion beam (FIB) milling through the gold film and membrane. The sample is prepared on a separate membrane that requires no further processing, as long as it is sufficiently transparent for the reference beams. This greatly simplifies the fabrication process, as the milling of the object hole has no longer to be stopped just at the gold/membrane interface. In addition, the membrane with the optical elements can be used 


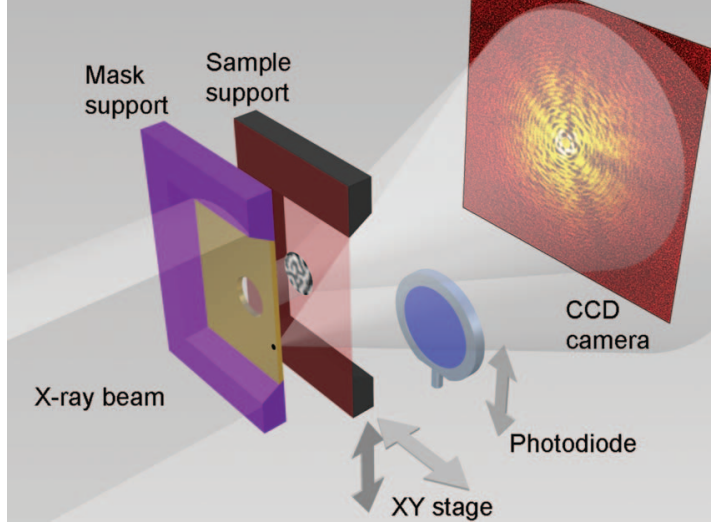

FIG. 1. (Color) Schematic of the x-ray holographic microscopy setup. It consists of a holography mask support, a movable sample support, and a CCD detector. The membrane with the optical elements (mask), i.e., the object and reference holes, is fixed in the center of the x-ray beam from the synchrotron (left side of the drawing). A second membrane, which is the support for the sample, can be moved freely in a plane perpendicular to the beam.

repeatedly for various samples. The sample membrane can be positioned by a motorized stage in the plane parallel to the optics membrane, while the latter is fixed in position with respect to the $\mathrm{x}$-ray beam. The two membranes are positioned in close contact but without exerting force, which allows for frictionless scanning. The need for coherent illumination of the scattering volume puts a restriction on the maximum allowed separation distance $L$ between FTH mask and sample. The path length difference between the scattered and the reference wave increases linearly with $L$, and we estimate $^{12}$ from the longitudinal coherence length of $1.6 \mu \mathrm{m}$ that $L$ must be kept smaller than $2 \mathrm{~mm}$ for effective interference. A photodiode can be inserted behind the membranes into the transmitted beam. It allows to measure absorption maps and spectra from the sample. As the contribution from the reference holes is small, the resolution of maps recorded with the diode by scanning the sample is of the size of the object hole. A $1.7 \mathrm{Mp}$ charge coupled device (CCD) camera (pixel size $20 \times 20 \mu \mathrm{m}^{2}$ ), which is protected by a central beam-stop, follows downstream at a distance of $360 \mathrm{~mm}$ from the sample. The experiments were carried out with circularly polarized light from the ID08 beamline at the ESRF. A pinhole with a diameter of $50 \mu \mathrm{m}$ was placed upstream of the sample in order to obtain a collimated and transversely partially coherent beam.

To demonstrate the capabilities of XHM, we investigate the magnetic domain structure of an exchange-coupled multilayer film with lateral parameter variation. A $\left(\mathrm{Co}_{0.7 \mathrm{~nm}} / \mathrm{Pt}_{2} \mathrm{~nm}\right)_{8}$ film was deposited by dc magnetronsputtering onto a $5 \mathrm{~nm}$ platinum seed layer. ${ }^{13,14}$ The multilayer has an out-of-plane easy axis of magnetization, which was confirmed using the magneto-optic Kerr effect. The film structure is tuned such that the multilayer is close to the parameters where spin reorientation will appear, ${ }^{14}$ i.e., where the magnetization will switch from an out-of-plane to an in-plane orientation. An iron wedge ranging from 0 to 4 $\mathrm{nm}$ in thickness was deposited on top of the last $2 \mathrm{~nm}$ thick Pt layer [Fig. 2(a)] and protected by a final $2 \mathrm{~nm}$ cap layer of platinum.

To localize the position of the wedge, we performed line scans of the sample with respect to the FTH mask along the

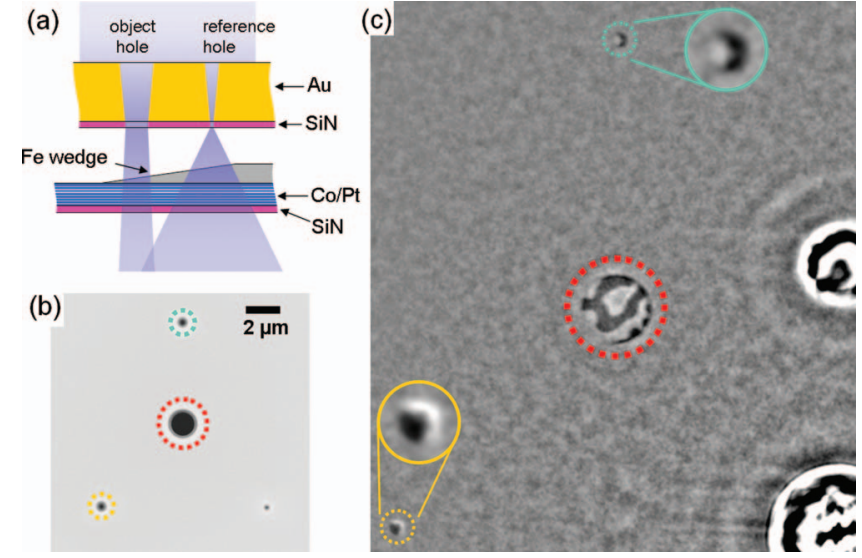

FIG. 2. (Color) Sample geometry and reconstruction of a single magnetic domain image. A cut through the scattering plane is shown in (a). The sample is illuminated through the optics membrane [SEM micrograph (b)]. Image (c) is a cutout of the real part of the FFT reconstruction of the difference of two holograms acquired with opposite helicities at the cobalt $L_{3}$ absorption edge. The autocorrelation of the object hole is located at the lower right corner. The correlation of the object hole (center, marked red) and the two larger reference holes (yellow and green, magnified in the insets) with the small reference hole yield the magnetic domain patterns at the respective positions.

$x$ - and $y$-directions with the photon energy tuned to the iron $L_{3}$ absorption edge $(707 \mathrm{eV})$. From the photodiode current, a normalized absorption profile along the wedge has been obtained, as shown in Fig. 3(a). It can be used to calculate the local iron coverage using the white line absorption length of $18 \mathrm{~nm}$ for iron. ${ }^{15}$ Magnetic images were obtained using the $\mathrm{XMCD}$ at the cobalt $L_{3}$ absorption edge $(778 \mathrm{eV})$. The diameter of the object hole was $1.5 \mu \mathrm{m}$. It was surrounded by three reference holes, placed on a circle with radius $7 \mu \mathrm{m}$ [Fig. 2(b)]. The reference hole used for image reconstruction had a diameter of $115 \mathrm{~nm}$, while two larger holes sized 300 $\mathrm{nm}$ were also present. At each location along the wedge, two holograms were acquired with opposite photon helicities. The acquisition time per image was $1.4 \mathrm{~s}$, and 100 images for
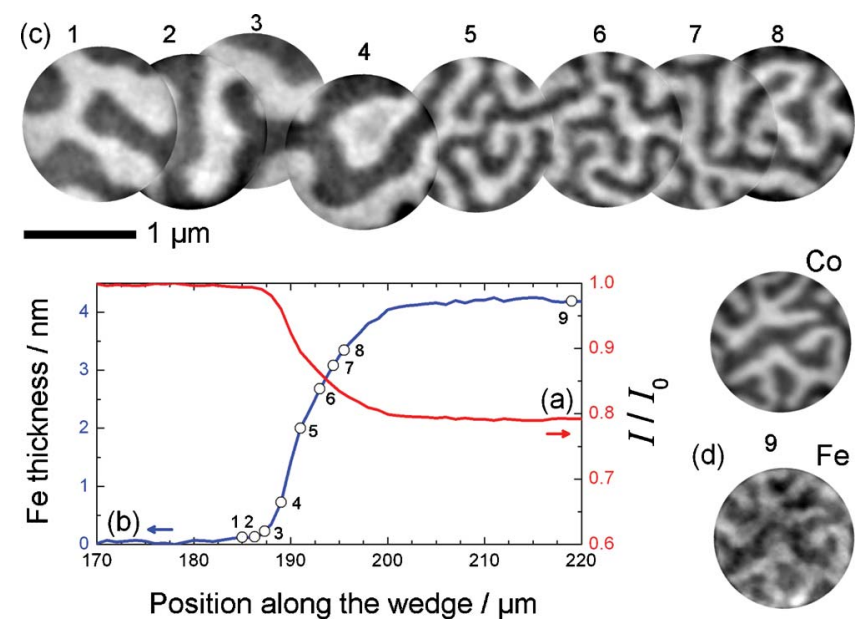

FIG. 3. (Color online) Domain size evolution of a $\mathrm{Co} / \mathrm{Pt}$ multilayer film covered by an iron wedge. Plot (a) gives the absorption profile (normalized photodiode current) at the Fe $L_{3}$ absorption edge when scanning over the $\mathrm{Fe}$ wedge. The absorption is used to calculate the local iron overlayer thickness (b). A contiguous series of XMCD holograms at the Co $L_{3}$ absorption edge has been acquired and reconstructed (c) at the indexed positions along the iron wedge. Image (d) has been measured at the $\mathrm{Fe} L_{3}$ absorption edge at the very same position as the last Co image (\#9). 
each helicity have been accumulated for better statistics. To obtain a real-space reconstruction of the magnetic domain pattern, a reverse Fourier transform is applied to the difference of both images, ${ }^{16}$ an example of which is shown in Fig. 2 (c). The series of domain images extracted from the reconstructed holograms [Fig. 3(c)] reveal a strong magnetic contrast with good signal-to-noise ratio. The total imaged area is $7.5 \mu \mathrm{m} \times 1.5 \mu \mathrm{m}$, covering the extension of the wedge. The maximum scan area is limited by the size of the membrane only.

The images reveal that the iron coverage influences the remanent magnetic state of the $\mathrm{Co} / \mathrm{Pt}$ multilayer. With increasing iron thickness, the average domain size of the cobalt layers shrinks from $280 \mathrm{~nm}$ without to $140 \mathrm{~nm}$ at $4 \mathrm{~nm}$ iron layer thickness, as has been deduced from a geometrical analysis of the reconstructed image series. The driving force for domains in magnetic thin films with out-of-plane easy axis is the dipolar energy, which can be lowered by an alternating up and down magnetization arrangement. This reduction is counterbalanced by an increase in domain wall energy corresponding to a larger total wall length with decreasing domain size, so a stable equilibrium domain size is obtained. The addition of an iron overlayer to the multilayer changes the domain size and causes a shift toward smaller domains. This is an indication for parallel coupling between $\mathrm{Fe}$ and the topmost Co layer, which adds to the dipolar energy of the system. Therefore, at equilibrium, a larger total domain wall length can be sustained, which is equivalent to a smaller domain size.

Tuning the x-ray photon energy to the iron $L_{3}$ absorption edge allows to image the magnetic domains of the iron wedge. Figure 3(d) shows such a reconstructed iron image at $4 \mathrm{~nm}$ thickness that was acquired at the same position as the cobalt image \#9. Both show an identical domain pattern, indicating a parallel orientation of the probed out-of-plane magnetization components of the iron film and the cobalt layers. Thus, the exchange interaction between the iron film and the multilayer-stack favors ferromagnetic coupling across the platinum layer, which is consistent with our interpretation of the domain size decrease. The strong dichroic contrast of the images at the cobalt absorption edge, especially when compared to the contrast of the image at the iron edge, suggests parallel coupling of all cobalt layers in the multilayer stack.

Good magnetic images are obtained although the reference beam is traversing the ferromagnetic material. The main effect here is additional absorption, which reduces the reference wave intensity. At the cobalt $L_{3}$ absorption edge, we estimate a transmission of $25 \%$ for the total multilayer stack, taking into account a magnetization averaged white line absorption length of $18 \mathrm{~nm}$ for cobalt. ${ }^{15}$ Since the reference beam passes through magnetic material, additional magnetic dichroism has to be expected. We observe this effect in the reconstructed images at the cross correlation positions of the small reference hole with the two larger ones. At different sample positions, these regions of the images appear either bright or dark and some times even split [Fig. 2(c)], when a large reference hole is by chance positioned above a domain wall. The influence of this effect on the reconstructed image is a global factor on the magnetic contrast, so the spatial magnetic information is in general not disturbed.

We have demonstrated a holographic microscopy method where the separation of the optical elements from the sample gives access to a new class of experiments with the possibility of arbitrary sample shift. The magnetic structure of a semitransparent multilayer film could be investigated as function of lateral thickness variation. Our design provides high flexibility because an easy sample exchange is possible without altering the optical properties of the setup. Key benefit is still the presence of a reference wave, which allows for unambiguous image reconstruction, as compared to plain CDI techniques. This concept has the potential for use at synchrotron sources as self-contained microscope, where the users can bring their samples on standard commercial membranes without the need for individual FIB processing.

Funding by DFG within SFB 668, as well as by BMBF and Free and Hanseatic City of Hamburg via Grant No. HBFG1021-627 and within the "Landesexzellenzinitiative Hamburg" is acknowledged. We thank the ESRF staff, in particular R. Barrett for engineering support, G. Retout, T. Trenit, and R. Homs-Regojo for technical assistance.

${ }^{1}$ J. Miao, P. Charalambous, J. Kirz, and D. Sayre, Nature (London) 400, 342 (1999).

${ }^{2}$ P. Thibault, M. Dierolf, A. Menzel, O. Bunk, C. David, and F. Pfeiffer, Science 321, 379 (2008).

${ }^{3}$ B. Abbey, K. A. Nugent, G. J. Williams, J. N. Clark, A. G. Peele, M. A. Pfeifer, M. de Jonge, and I. McNulty, Nat. Phys. 4, 394 (2008).

${ }^{4}$ S. Eisebitt, J. Lüning, W. F. Schlotter, M. Lörgen, O. Hellwig, W. Eberhardt, and J. Stöhr, Nature (London) 432, 885 (2004).

${ }^{5}$ E. Guehrs, C. M. Günther, R. Könnecke, B. Pfau, and S. Eisebitt, Opt. Express 17, 6710 (2009).

${ }^{6}$ L.-M. Stadler, C. Gutt, T. Autenrieth, O. Leupold, S. Rehbein, Y. Chushkin, and G. Grübel, Phys. Rev. Lett. 100, 245503 (2008).

${ }^{7}$ C. M. Günther, F. Radu, A. Menzel, S. Eisebitt, W. F. Schlotter, R. Rick, J. Lüning, and O. Hellwig, Appl. Phys. Lett. 93, 072505 (2008); A. Scherz, W. F. Schlotter, K. Chen, R. Rick, J. Stöhr, J. Lüning, I. McNulty, C. Günther, F. Radu, W. Eberhardt, O. Hellwig, and S. Eisebitt, Phys. Rev. B 76, 214410 (2007); T. Hauet, C. M. Günther, B. Pfau, M. E. Schabes, J.-U. Thiele, R. L. Rick, P. Fischer, S. Eisebitt, and O. Hellwig, ibid. 77, 184421 (2008).

${ }^{8}$ A. Scherz, D. Zhu, R. Rick, W. F. Schlotter, S. Roy, J. Lüning, and J. Stöhr, Phys. Rev. Lett. 101, 076101 (2008).

${ }^{9}$ W. F. Schlotter, R. Rick, K. Chen, A. Scherz, J. Stöhr, J. Lüning, S. Eisebitt, C. Günther, W. Eberhardt, O. Hellwig, and I. McNulty, Appl. Phys. Lett. 89, 163112 (2006).

${ }^{10}$ S. Marchesini, S. Boutet, A. E. Sakdinawat, M. J. Bogan, S. Bajt, A. Barty, H. N. Chapman, M. Frank, S. P. Hau-Riege, A. Szoke, C. Cui, D. A. Shapiro, M. R. Howells, J. C. H. Spence, J. W. Shaevitz, J. Y. Lee, J. Hajdu, and M. M. Seibert, Nat. Photonics 2, 560 (2008).

${ }^{11}$ R. Rick, A. Scherz, W. F. Schlotter, D. Zhu, J. Lüning, and J. Stöhr, Opt. Lett. 34, 650 (2009).

${ }^{12}$ D. L. Abernathy, G. Grübel, S. Brauer, I. McNulty, G. B. Stephenson, S. G. J. Mochrie, A. R. Sandy, N. Mulders, and M. Sutton, J. Synchrotron Radiat. 5, 37 (1998).

${ }^{13}$ M. Wellhöfer, M. Weissenborn, R. Anton, S. Pütter, and H. P. Oepen, J. Magn. Magn. Mater. 292, 345 (2005).

${ }^{14}$ H. Stillrich, C. Menk, R. Frömter, and H. P. Oepen, J. Appl. Phys. 105, 07C308 (2009).

${ }^{15}$ R. Nakajima, J. Stöhr, and Y. U. Idzerda, Phys. Rev. B 59, 6421 (1999).

${ }^{16}$ S. Streit-Nierobisch, D. Stickler, C. Gutt, L.-M. Stadler, H. Stillrich, C. Menk, R. Frömter, C. Tieg, O. Leupold, H. P. Oepen, and G. Grübel, J. Appl. Phys. 106, 083909 (2009). 